\title{
Faceting of Nanocrystals during Chemical Transformation: From Solid Silver Spheres to Hollow Gold Octahedra
}

\author{
Yadong Yin, ${ }^{1,2 \dagger}$ Can Erdonmez, ${ }^{2,3}$ A. Paul. Alivisatos ${ }^{1,2,3 *}$ \\ ${ }^{I}$ The Molecular Foundry and ${ }^{2}$ Materials Science Division at the Lawrence Berkeley National Laboratory, ${ }^{3}$ Department \\ of Chemistry, University of California at Berkeley, Berkeley, CA 94720, USA.
}

RECEIVED DATE (automatically inserted by publisher); Email: alivis@berkeley.edu

Sustained progress in nanocrystal synthesis has enabled recent use of these materials as inorganic, macromolecular precursors that can be chemically transformed into new nanostructures. ${ }^{1,2}$ The literature now contains several cases with chemical transformations being accompanied by varying degrees of modification of properties, including crystal structure and particle shape..$^{3-5}$ As a recent example, we demonstrated that as-synthesized metallic nanocrystals yield, upon oxidation, nanostructures with modified morphologies such as hollow particles. ${ }^{6}$ This morphological change derives from directional material flows due to differing diffusivities for the reacting atomic species, in a nanoscale version of the well-known Kirkendall Effect. This general methodology has since been extended by other groups to produce nanostructures with various compositions and shapes. $^{7-10}$

Galvanic replacement reactions have been demonstrated to also produce hollow nanostructures. Xia et al. reported cases where a replacement reaction took place uniformly around Ag cubes of $\sim 100 \mathrm{~nm}$ size, leading to formation of $\mathrm{Au}$ nanoboxes. The exterior shape of the nanoboxes tends to largely reproduce that of the sacrificial $\mathrm{Ag}$ counterparts. ${ }^{11-15}$ Here, we report that performing the same replacement reaction on silver nanocrystals that are an order of magnitude smaller leads to significant changes in the external morphology of the $\mathrm{Au}$ shells as the reaction proceeds, while still creating a central void in each particle. Specifically, single crystalline silver nanoscrystals with a spherical shape act in the presence of $\mathrm{Au}^{3+}$ as precursors for formation of hollow $\mathrm{Au}$ nanocrystals with truncated octahedral shape. The growth of significantly faceted particles from spherical precursors is made possible by the enhanced role of surface effects in our smaller nanocrystals. Production of hollow Au nanocrystals with faceted geometry allows increased tunability of optical properties as the surface plasmon resonance spectra of a hollow metallic nanocrystal depends strongly not only on the shell thickness, but also on the detailed shape. ${ }^{16,17}$

To produce $\mathrm{Ag}$ nanocrystals with reduced sizes and improved monodispersity, we performed the synthesis using a modification of the polyol process in an organic solvent and at high temperature. ${ }^{6,18}$ The silver salt $\mathrm{AgNO}_{3}$ was reduced by a long chain polyol such as 1,2hexadecanediol using an organic solvent, o-dichlorobenzene (DCB). Oleylamine was present as a surfactant. Near instant formation of silver nanocrystals upon reaction was indicated by the originally colorless solution turning dark brown. The transformation of solid nanocrystals into hollow ones was performed through galvanic replacement by dropwise addition of gold (III) chloride solution to diluted silver colloidal solution until the solution changed in color from dark yellow to blue. In this reaction, oleylamine likely serves two purposes. First, it solubilizes the precursors $\mathrm{AgNO}_{3}$ and $\mathrm{AuCl}_{3}$ in $\mathrm{DCB}$. Second, it acts as a surfactant that controls the growth of nanocrystals and stabilizes the colloidal solution.

The choice of DCB as the solvent allows us to synthesize silver nanocrystals at relatively high temperatures $\left(\sim 180^{\circ} \mathrm{C}\right)$. The resulting high nucleation and growth rates make "kinetic size focusing" possible, favoring formation of monodisperse nanocrystals. ${ }^{1,}{ }^{19}$ Indeed, assynthesized silver nanocrystals have narrow size distributions, as seen in the transmission electron microscopy (TEM) image and the observed particle diameter statistics for a typical sample (Fig 1A). All the nanocrystals possess an apparently spherical shape and the average diameter is $11.0 \pm 1.1 \mathrm{~nm}$. Average nanoparticle size can be tuned by varying reaction conditions such as the growth duration, temperature, and the molar ratio between the surfactant and silver precursor. ${ }^{20} \mathrm{We}$ also notice that the nanocrystals synthesized in this case have average sizes much smaller than those produced using the regular polyol process which typically uses ethylene glycol as solvent as well as reducing agent, and

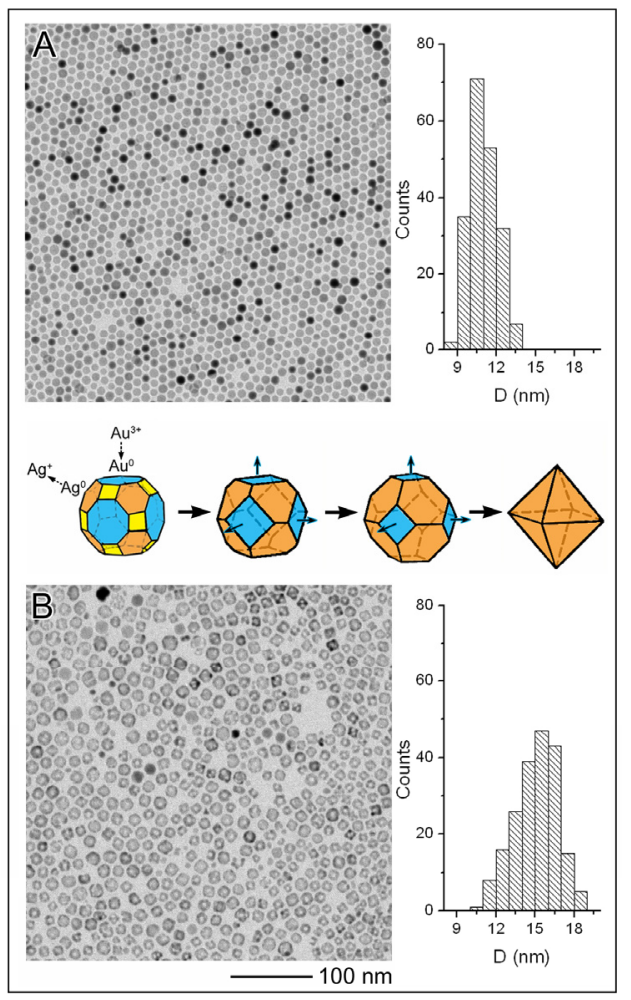

Figure 1. (A) TEM image of Ag nanocrystals and the measured particle diameter distribution. (B) TEM image and diameter distribution for Au hollow nanocrystals obtained by performing a replacement reaction on the Ag nanocrystals in (A). The schematic illustration between (A) and (B) shows the proposed mechanism for the formation of $\mathrm{Au}$ truncated octahedra. A truncated cuboctahedron is used to represent the starting spherical $\mathrm{Ag}$ nanocrystal. A replacement reaction between $\mathrm{Au}$ and $\mathrm{Ag}$ removes $\mathrm{Ag}$ atoms preferentially from (111) facets, while depositing $\mathrm{Au}$ atoms selectively to higher energy facets such as (100) and (110).

polyvinylpyrrolidone (PVP) as surfactant. ${ }^{21,22}$ This suggests stronger attachment of oleylamine than PVP to the silver nanocrystal surface.

Performing a replacement reaction on Ag nanocrystals with dissolved $\mathrm{Au}^{3+}$ leads to formation of hollow gold nanocrystals with slightly broadened size distribution, as shown in Fig 1B. Contrast is sensitive to mass-thickness in conventional TEM images; consequently, hollow particles appear lighter at their center in images. Energy Dispersive X-ray analysis (EDX) on clusters of Au hollow nanocrystals typically does not give rise to a silver signal, implying that the replacement reaction proceeds essentially to completion and that $\mathrm{Ag}$ is present at $<5 \%$, if at all, in these nanocrystals.

Close inspection of the hollow nanocrystals reveals that they have faceted morphology instead of the spherical shape of the original Ag particles, with some of them clearly showing a rhomboid shape. As the faceted nanocrystals are viewed along various projections in TEM images, the size dispersion of the sample is difficult to characterize exactly. Measuring the longest dimension observed in images of individual hollow

\footnotetext{
${ }^{\dagger}$ Current address: Department of Chemistry, University of California at Riverside, Riverside, CA 92521. Email: yadong.yin@ucr.edu
} 


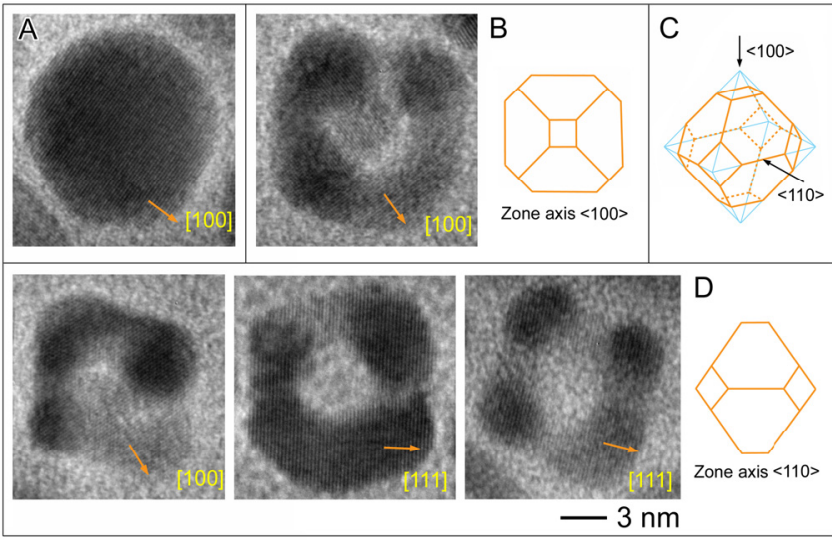

Figure 2. (A) HRTEM image of a $\mathrm{Ag}$ nanocrystal, confirming spherical morphology and single crystalline structure. (B-D) HRTEM images of Au hollow nanocrystals, showing a faceted morphology and a single crystalline domain within each particle: (B) imaged along $<100>$ direction; (D) imaged along $<110>$ direction. Various views of the faceted nanocrystals are consistent with a truncated octahedron as the dominant particle shape, as illustrated schematically.

crystals yields an average length of $15.1 \pm 1.7 \mathrm{~nm}$, a number significantly larger than that of the starting $\mathrm{Ag}$ particles. The increase in length can not be attributed to systematic errors during size measurements; instead, it indicates that the particles grow outwards along certain directions during the replacement reaction.

Fig. 2A shows the high resolution TEM (HRTEM) image of a Ag nanocrystal before the replacement reaction. Lattice fringes are separated by $2.04 \AA$ corresponding to $\{200\}$ lattice planes of face-centered cubic $(f c c)$ Ag. Unlike the regular polyol process where Ag nanocrystals usually contain defects such as multiple twins, ${ }^{11,} 12,23$ our modified method produces only single crystalline Ag particles with spherical shapes. The hollow nanocrystals obtained by the replacement reaction retain the single crystalline structure despite significant shape change. The various profiles observed in images of hollow nanocrystals can be shown to correspond to various projections of a truncated octahedral morphology enclosed by six $\{100\}$ and eight $\{111\}$ facets shown schematically in Fig. 2C. The truncated octahedral shape has been found previously for solid nanocrystals of noble metals such as silver and gold. ${ }^{24}$ Figs. $2 \mathrm{~B}$ and $2 \mathrm{D}$ show HRTEM images and schematic illustrations of how viewing of nanocrystals along particular directions accounts for the different images. When a nanocrystal lies on one of its $\{100\}$ facets, the projected image is a square with four corners slightly cut off. The extent of truncation of the octahedral particle determines the degree the corners are cut off in the projected square. Fig. 2B shows a representative HRTEM image of a hollow nanocrystal positioned on its $\{100\}$ facet. The spacing between the fringes is $\sim 2.04 \AA$ in agreement with the $\{200\}$ lattice spacing of $f c c \mathrm{Au}$. Observed orientation of these $\{200\}$ fringes parallel to square diagonals is expected for the case of the six truncated octahedral vertices lying along the six $<100>$ directions and imaged along one of the $<100>$ directions. Fig. 2D shows the case when a nanocrystal sits on one of the edges formed between two $\{111\}$ facets. As illustrated in the model, the projection is along $<110\rangle$ and the particle has a rhomboid shape with two corners cut off. HRTEM analyses indicate that both $\{200\}$ and $\{111\}$ can appear, depending on the imaging conditions. The fringes corresponding to $\{200\}$ planes are parallel to the short diagonal of the rhombus, while those corresponding to $\{111\}$ planes are parallel to the edges of the rhombus. Based on these observations, we conclude that the spherical $\mathrm{Ag}$ nanocrystals have been transformed into hollow, truncated octahedra. The degree of truncation varies from particle to particle; typically, the size of a corner facet is small in comparison to the edge length of the octahedron.

The shape transformation from $\mathrm{Ag}$ nanospheres to $\mathrm{Au}$ truncated octahedra involves a number of processes including the oxidation and dissolution of $\mathrm{Ag}$ atoms, reduction and deposition of $\mathrm{Au}$ atoms, and counterdiffusion of vacancies and atoms. It is not yet possible to detail an exact mechanism, but reasonable arguments may be made about the formation process. The generally observed trend for surface energies of the low-index faces for noble metal crystals seems to be $\gamma_{111}<\gamma_{100}<\gamma_{110}{ }^{23}$ For our Ag synthesis conditions, the apparently spherical shape of the resulting particles indicates that several different families of slow-growing facets grow at a similar rate. When silver nanocrystals are subjected to the replacement reaction, the $\mathrm{Au}^{3+}$ cations are reduced and deposited on the surface of Ag nanocrystals. Net growth or etching of each facet during the reaction is determined by the competition between $\mathrm{Au}$ atom deposition and Ag atom dissolution occurring at that facet. Our results are consistent with $\mathrm{Au}$ deposition occurring preferentially onto the high energy $\{100\}$ and $\{110\}$ facets, and Ag dissolution occurring preferentially at the $\{111\}$ facets. In an idealized picture, release of three $\mathrm{Ag}$ atoms from the nanocrystal for each $\mathrm{Au}$ atom deposited injects vacancies at the core-shell interface. Similar to the case of sulfidation of cobalt nanocrystals, a large fraction of these vacancies coalesce, producing a well-defined void in the center of each nanocrystal. ${ }^{6}$ In previous studies, pinholes were observed to form in the $\mathrm{Au}$ shells at later stages of the replacement reaction. ${ }^{22} \mathrm{We}$ also observe pinholes and they appear preferentially on $\{111\}$ facets of the hollow particles (supporting information), consistent with the argument that $\mathrm{Au}$ deposition is not preferred on these facets during the replacement reaction.

Evolution of nanocrystal shape towards an octahedral geometry during the replacement reaction is very visible in comparison to the results by Xia et al. Effectively, the net growth rate on $\{100\}$ faces exceeds significantly net growth rate on $\{111\}$ faces for our smaller, spherical nanocrystals; this is not the case for the $100 \mathrm{~nm}$ nanoboxes, except very close to completion of the reaction, when slight faceting of cube corners become visible. Apparently, differences in growth and/or etching rate among various crystal facets become enhanced as particle size decreases.

Consistent with previous studies, the surface plasmon peak is dramatically red shifted for hollow metallic nanocrystals in comparison to solid counterparts (supporting information). A diluted solution of the starting silver nanocrystals is yellow; as the reaction proceeds, it progressively turns brownish yellow, greenish yellow, and eventually blue. Currently, we are performing spectroscopic studies on single particles to identify how surface plasmon resonance properties depend on combined presence of central voids and a variable degree of faceting for the hollow shells.

In summary, we have demonstrated that a replacement reaction performed on single crystalline silver nanospheres in an organic solvent produces hollow gold nanocrystals with faceted shape, while preserving the single crystalline nature of the starting particles. This study reveals that surface-mediated shape control and achievement of a hollow morphology can be combined in a one-pot, single-step synthetic procedure, leading to finer tuning of particle morphology. As various phenomena of deposition, etching and void formation are coupled within the synthetic process, detailed mechanistic studies will be necessary for further progress along this direction.

Acknowledgement. This work was performed at the Molecular Foundry, Materials Science Division, Lawrence Berkeley National Laboratory, and was supported by the Office of Science, Office of Basic Energy Sciences, of the U.S. Department of Energy under Contract No. DE-AC02-05CH11231. Y.Y. thanks Shaul Aloni at the Molecular Foundry for help with the EDX measurements.

Supporting Information Available: Experimental procedures, additional HRTEM analysis and UV-Vis measurements. This material is available free of charge via the Internet at http://pubs.acs.org.

(1) Yin, Y.; Alivisatos, A. P. Nature 2005, 437, 664-670.

(2) Stellacci, F. Nat. Mater. 2005, 4, 113-114

(3) Harris, P. J. F. Nature 1986, 323, 792-794

(4) Son, D. H.; Hughes, S. M.; Yin, Y.; Alivisatos, A. P. Science 2004, 306 , 1009-1012.

(5) Mokari, T.; Rothenberg, E.; Popov, I.; Costi, R.; Banin, U. Science 2004, $304,1787-1790$.

(6) Yin, Y.; Rioux, R. M.; Erdonmez, C. K.; Hughes, S.; Somorjai, G. A.; Alivisatos, A. P. Science 2004, 304, 711-714.

(7) Chang, Y.; Teo, J. J.; Zeng, H. C. Langmuir 2005, 21, 1074-1079.

(8) Liu, B.; Zeng, H. C. J. Am. Chem. Soc. 2004, 126, 16744 - 16746.

(9) Wang, C. M.; Baer, D. R.; Thomas, L. E.; Amonette, J. E.; Anthony, J.; Qiang, Y.; Duscher, G. J. Appl. Phys. 2005, 98, 094308.

(10) Li, Q.; Penner, R. M. Nano Lett. 2005, 5, $1720-1725$.

(11) Sun, Y.; Mayers, B. T.; Xia, Y. Nano Lett. 2002, 2, 481-485.

(12) Sun, Y.; Xia, Y. Science 2002, 298, 2176-2179.

(13) Liang, H.-P.; Zhang, H.-M.; Hu, J.-S.; Guo, Y.-G.; Wan, L.-J.; Bai, C.-L. Angew. Chem. Int. Ed. 2004, 43, 1540-1543.

(14) Selvakannan, P.; Sastry, M. Chem. Comm. 2005, 1684-1686.

(15) Sun, Y.; Mayers, B.; Xia, Y. Adv. Mater. 2003, 15, 641-646.

(16) Halas, N. MRS Bull. 2005, 30, 362-367.

(17) Haes, A. J.; Haynes, C. L.; McFarland, A. D.; Schatz, G. C.; Van Duyne, R. P.; Zou, S. MRS Bull. 2005, 30, 368-375.

(18) Sobal, N. S.; Ebels, U.; Möhwald, H.; Giersig, M. J. Phys. Chem. B 2003, 107, 7351-7354.

(19) Peng, X.; Wickham, J.; Alivisatos, A. P. J. Am. Chem. Soc. 1998, 120 $5343-5344$

(20) Lin, X. Z.; Teng, X.; Yang, H. Langmuir 2003, 19, 10081-10085.

(21) Fievet, F.; Lagier, J. P.; Figlarz, M. MRS Bull. 1989, 14, 29-34.

(22) Sun, Y.; Xia, Y. Nano Lett. 2003, 3, 1569-1572.

(23) Wang, Z. L. J. Phys. Chem. B 2000, 104, 1153-1175.

(24) Harfenist, S. A.; Wang, Z. L.; Alvarez, M. M.; Vezmar, I.; Whetten, R. L. J. Phys. Chem. 1996, 100, 13904-13910. 


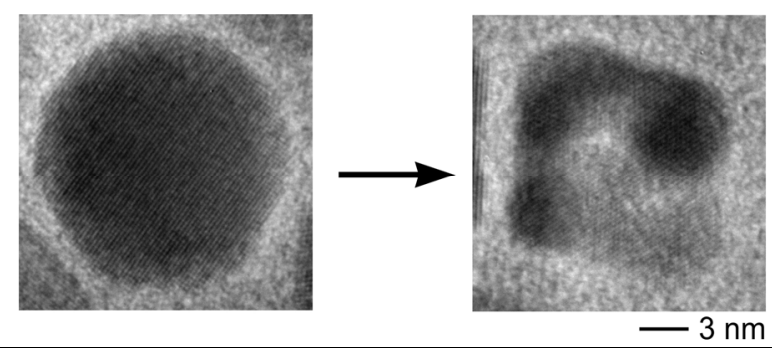

We demonstrate that performing a replacement reaction on single crystalline $\mathrm{Ag}$ nanospheres of $\sim 10 \mathrm{~nm}$ in diameter in an organic solvent produces hollow Au nanocrystals with an octahedral shape. Different from those Au shells made by starting with $\mathrm{Ag}$ particles about one order of magnitude larger, which largely reproduce that of the sacrificial Ag counterparts, the hollow nanocrystals obtained in this work show significant changes in the external morphology from the spherical Ag precursors. This evolution of a faceted external morphology during chemical transformation is made possible by the enhanced role of surface effects in our smaller nanocrystals. The competition between the Au atom deposition and Ag atom dissolution on various nanocrystal surfaces is believed to determine the final octahedral shape of the hollow Au nanocrystals. Simultaneous achievement of surface-mediated shape control and a hollow morphology in a one-pot, single-step synthetic procedure in this study promises an avenue to finer tuning of particle morphology, and thus physical properties such as surface plasmon resonance. 